\title{
ESPERANÇA DE FAMÍLIAS QUE CONVIVEM COM COMPORTAMENTO ADITIVO POR TEMPO PROLONGADO
}

\section{HOPE OF FAMILIES LIVING WITH LONG-TERM ADDITIVE BEHAVIOR}

\section{ESPERANZA DE FAMILIAS QUE CONVIVEN CON COMPORTAMIENTO ADITIVO POR TIEMPO PROLONGADO}

Indianatha de Kássia Santana Elvira ${ }^{1}$, Lúcia Margarete dos Reis ${ }^{2}$, Aroldo Gavioli ${ }^{3}$, Sonia Silva Marcon ${ }^{4}$, Magda Lúcia Félix de Oliveira ${ }^{5}$.

\section{RESUMO}

Objetivo: Identificar a esperança de famílias que convivem por tempo prolongado com o comportamento aditivo de um de seus membros. Métodos: Estudo transversal com familiares de 29 indivíduos internados com trauma físico associado à intoxicação por drogas de abuso, considerados eventos sentinela em um programa de vigilância epidemiológica das repercussões do uso de drogas em famílias. Foram utilizados um roteiro semiestruturado de entrevista e a Escala de Hopefulness-hopelessness. $O$ teste de MannWhitney foi empregado para se verificar associações estatísticas. Resultados: Os usuários tinham, em média, idade de 40,1 anos e tempo médio de uso de drogas de 20,8 anos. $O$ escore médio de esperança dos familiares foi de $28,81( \pm 2,83)$. A idade, o tempo de uso e o poliuso de drogas pelos usuários foram estatisticamente associados aos menores níveis de esperança, enquanto a prática religiosa, pelos familiares, foi estatisticamente associada a maiores níveis de esperança. Conclusão: Observou-se um escore médio de esperança baixo, indicando a necessidade de intervenção sobre esse sentimento nas famílias estudadas.

Descritores: Drogas llícitas; Alcoolismo; Expectativa de Vida; Relações Familiares; Saúde da Família.

\begin{abstract}
Objective: To identify the hope of families living for an extended period with the additive behavior of one of their family members. Methods: A retrospective and cross-sectional study was conducted with relatives of 29 hospitalized individuals with physical trauma associated with drug intoxication, considered as sentinel events in a program of epidemiological surveillance of the repercussions of drug use in families. It was used a semi-structured interview script and the Hopefulness-hopelessness Scale. The Mann-Whitney test was used to verify statistical associations. Results: Users had a mean age of 40.1 years and mean time of drug use of 20.8 years. The mean family hope score was 28.81 ( \pm 2.83 ). Age, time of use and use of many drugs by users were statistically associated with lower levels of hope, while religious practice by relatives was statistically associated with higher levels of hope. Conclusion: A mean score of low expectation was observed, indicating the need for intervention on this feeling in the families studied.
\end{abstract}

Descriptors: Street Drugs; Alcoholism; Life Expectancy; Family Relations; Family Health.

\section{RESUMEN}

Objetivo: Identificar la esperanza de familias que conviven por tiempo prolongado con el comportamiento aditivo de uno de sus miembros. Métodos: Estudio retrospectivo y transversal, con familiares de 29 personas internadas con trauma físico asociado a la intoxicación por drogas de abuso, considerados eventos centinela en un programa de vigilancia epidemiológica de las repercusiones del uso de drogas en familias. Se utilizó un guión semiestructurado de entrevista y la Escala de Hopefulnesshopelessness. La prueba de Meann-Whitney se utilizó para comprobar las asociaciones estadísticas. Resultados: Los usuarios tenían un promedio de edad de 40,1 años y el tiempo promedio de uso de drogas de 20,8 años. El puntaje medio de esperanza de los familiares fue de $28,81( \pm 2,83)$. La edad, el tiempo de consumo y el uso de muchas drogas por parte de los usuarios se asociaron estadísticamente con niveles más bajos de esperanza, mientras que la práctica religiosa de los familiares se asoció estadísticamente con níveles más altos de esperanza. Conclusión: Se observó un puntaje medio de esperanza bajo, indicando la necesidad de intervención sobre ese sentimiento en las familias estudiadas.

Descriptores: Drogas llícitas; Alcoholismo; Esperanza de Vida; Relaciones Familiares; Salud de la Familia.

\footnotetext{
${ }^{1}$ Mestranda em Enfermagem do Programa de Pós-Graduação em Enfermagem da Universidade Estadual de Maringá. Enfermeira intervencionista de Urgência e Emergência do Samu Norte Novo/Maringá. ${ }^{2}$ Doutora em Enfermagem pela Universidade Estadual de Maringá. ${ }^{3}$ Mestre em Enfermagem, Discente do Curso de Doutorado em Enfermagem da Universidade Estadual de Maringá. ${ }^{4}$ Doutora em Filosofia da Enfermagem. Docente da Universidade Estadual de Maringá e do Programa de PósGraduação em Enfermagem da Universidade Estadual de Maringá. ${ }^{5}$ Doutora em Saúde Coletiva. Docente da Universidade Estadual de Maringá e do Programa de PósGraduação em Enfermagem da Universidade Estadual de Maringá.
} 


\section{INTRODUÇÃO}

Estudos apontam que grande parcela da população mundial é afetada, direta ou indiretamente, pelo uso abusivo, comercialização e violência relacionados às drogas, com repercussões reconhecidas na saúde pessoal, na vida familiar, no trabalho e na comunidade de convivência do usuário ${ }^{(1-2)}$. Nessa perspectiva, o abuso de drogas deve ser discutido no campo de formação dos profissionais de enfermagem, principalmente sobre a utilização de metodologias assistenciais de promoção da saúde, prevenção, cuidado e reinserção social inclusiva à família ${ }^{(3)}$.

A família é considerada estratégica para a proteção e socialização de seus membros e para a transmissão dos valores sociais e culturais, além de exercer influência significativa sobre as crenças daqueles, por meio de atitudes e comportamentos relacionados ao processo saúde-doença para lidar com hábitos considerados não saudáveis no contexto familiar, como o abuso de drogas ${ }^{(1,4)}$.

Como cuidadora, afetiva, amorosa e comunicativa, a família tem importante papel na prevenção do uso de drogas e na promoção da resiliência, garantindo maiores chances de promover condições e possibilidades para o desenvolvimento saudável dos filhos. Porém, quando existem pais ou familiares usuários de álcool e outras drogas, bem como a presença de relações familiares disfuncionais, a família aparece também como um fator de risco para comportamentos que podem levar ao uso abusivo de álcool e de outras drogas ${ }^{(5)}$.

Dessa forma, a família pode tornar-se fator motivacional tanto para estimular a superação do uso de drogas como para negligenciar o esforço de quem está tentando se recuperar, oferecendo ainda mais razões para o uso ${ }^{(3,6)}$. Nesse contexto, qualquer programa de prevenção de uso de drogas necessita prever aplicações práticas de orientação familiar ${ }^{(5)}$.

Sobre as repercussões das drogas de abuso na família, a experiência dos familiares afetados pode ser devastadora nos aspectos psicológico, financeiro, social e interpessoal do funcionamento familiar. $\mathrm{O}$ uso de drogas por um membro da família causa grande pressão sobre o restante dos membros, o que ocasiona dificuldades para se lidar com o problema familiar e com conflitos relacionados ao uso de $\operatorname{drogas}^{(2-4,7)}$.
O impacto do uso de drogas na família se dá por meio de sentimentos negativos como tensão, estresse, preocupação, estigma, raiva e culpa $^{(2,3,7)}$, entretanto perseverança, coragem, otimismo e esperança são essenciais para se resistir às dificuldades acrescidas no cotidiano familiar $^{(3,6)}$.

A esperança é uma variável psicológica que se reveste de extrema importância para a vida humana e pode ser definida como uma probabilidade subjetiva de bons resultados, constituindo, para o homem, o sentimento de um futuro otimista, que lhe permita a elaboração de planos e objetivos em longo prazo ${ }^{(8,9)}$. Diante das situações do cotidiano, as pessoas tendem a ancorar sua esperança em questões que lhes são significativas, sejam elas externas, como família, amigos ou algo sobrenatural, como internas, quando depositam suas esperanças em si mesmas e na possibilidade de suas realizações ${ }^{(8)}$.

Pela importância da esperança no processo de enfrentamento de doenças, seus níveis vêm sendo explorados em indivíduos que vivenciam doenças crônicas e em seus cuidadores familiares, a fim de se verificar o impacto desse sentimento em momentos de dificuldades e provações ${ }^{(9)}$. A importância da esperança já foi investigada em pacientes com esquizofrenia ${ }^{(10)}$, com câncer em tratamento quimioterápico ${ }^{(11)}$ e em familiares cuidadores ${ }^{(9-10)}$.

No contexto brasileiro, a esperança de familiares de usuários de drogas, principalmente aqueles com longo período de convivência com as drogas no ambiente familiar, ainda é pouco investigada e, nesse sentido, o presente estudo se propõe a analisar a interface do fenômeno drogas de abuso e a esperança de famílias, tendo como objetivo identificar a esperança de famílias que convivem por tempo prolongado com o comportamento aditivo de um de seus membros.

\section{METODOLOGIA}

Estudo transversal, realizado no município de Maringá- PR, série de casos de familiares de usuários de drogas de abuso submetidos à metodologia de investigação epidemiológica de evento sentinela ${ }^{(11)}$. O conceito de sentinel events foi proposto por Rutstein e colaboradores como a ocorrência de doença, invalidez ou morte desnecessária e prevenível por ações da atenção primária, constituindo-se em clear-cuts, índices da qualidade da assistência à saúde de uso imediato ${ }^{(12)}$. Em nosso país, autores têm utilizado a ferramenta de evento sentinela como uma 
forma de "monitoramento de emergência" e defendem sua ampliação conceitual para qualquer manifestação de doença ou ocorrência que apresentem potencial para causar doenças, com a finalidade de avaliar aspectos específicos do processo de atenção à saúde ${ }^{(13-14)}$.

A amostra intencional de 29 familiares de usuários de drogas originou-se a partir da internação hospitalar de pessoas com diagnóstico médico de trauma físico associado à intoxicação por drogas de abuso, considerado evento sentinela em um programa para vigilância epidemiológica e monitoramento das repercussões do uso de drogas na saúde familiar e do acesso aos dispositivos de políticas públicas sobre drogas.

No presente estudo, os usuários de drogas eventos sentinela apresentavam quadro clínico compatível/sinais e sintomas sugestivos de intoxicação aguda ou crônica, ou foram submetidos a testes laboratoriais confirmatórios de intoxicação por drogas de abuso, tinham vínculo familiar e foram notificados ao Centro de Controle de Intoxicações do Hospital Universitário Regional de Maringá-PR, no período entre abril e setembro de 2014. Os familiares entrevistados cumpriram os critérios de inclusão: ser familiar dos eventos sentinela, ter idade superior a 18 anos e relação de convivência ou coabitação com o evento sentinela antes e após a internação deste. $O$ desfecho de óbito do evento sentinela foi utilizado como critério de exclusão.

A coleta de dados para o presente estudo foi realizada durante a segunda fase da investigação de cada evento sentinela, quando é feita uma entrevista com um familiar, e aconteceu em domicílio, em um único encontro; foram utilizados dois instrumentos de coleta de dados: roteiro para entrevista semiestruturada, composto de dois módulos: o primeiro de caracterização sociodemográfica dos participantes e padrão de uso das drogas dos eventos sentinelas e o segundo, composto da Escala de Hopefulness-hopelessness ${ }^{(15)}$.

Essa escala contém dez itens e é projetada para se avaliar o quão esperançoso o familiar se sente em relação ao futuro do seu familiar usuário de álcool e/ou drogas. Os dez itens são escritos na forma de afirmativas, apresentados no formato de escala do tipo Likert de 5 pontos, variando de concordo completamente, concordo, não sei, discordo e discordo completamente; os cinco primeiros itens da escala pontuam de 1 a 5 , correspondendo aos itens positivos, e os cinco últimos, a itens negativos, apresentando, portanto, escores invertidos. O escore total varia de 10 a 50 e, quanto maior o escore, mais alto é o nivel de esperança do familiar em relação ao futuro. A Escala de Hopefulness-hopelessness foi validada e traduzida para o português do Brasil em um estudo que avaliou a esperança em familiares de usuários de drogas ${ }^{(16)}$.

A análise dos dados foi realizada no Software Statistical Package for the Social Sciences SPSS ${ }^{\circledR}$ V. 24, e, para se avaliar a distribuição normal dos dados, foi utilizado o teste de Kolmogora-Smirnov, com correção de Liliefors, que não confirmou a normalidade dos dados, o que indicou a utilização de testes não paramétricos. Procedeu-se à análise descritiva dos dados por meio do cálculo de medidas de posição e dispersão, frequências absolutas e relativas. O Teste $U$ de Mann-Whitney foi empregado para se avaliar a associação das variáveis sociodemográficas; quanto ao escore de Esperança Hopefulness-hopelessness, considerou-se o nível de significância de p<0,05.

Os familiares foram informados e esclarecidos quanto à natureza da pesquisa e convidados a participar, assinando termo de consentimento livre e esclarecido. $O$ projeto foi aprovado pelo Comitê Permanente de Ética em Pesquisa Envolvendo Seres Humanos, da Universidade Estadual de Maringá, com parecer favorável no 458.185/2013.

\section{RESULTADOS E DISCUSSÃO}

Este estudo, que buscou mensurar a esperança de familiares que conviviam com usuários de drogas de abuso por tempo prolongado, tem como limitações o número reduzido de pacientes eventos sentinela, o fato de $o$ atendimento ter se iniciado em vista de traumas, o que dificulta a abordagem familiar, bem como sobre o instrumento utilizado, destacando-se sua pouca aplicação no contexto brasileiro, o que dificulta a comparação e a generalização dos resultados. Como fortalezas, destaca-se a possibilidade de se identificar a esperança de familiares de um grupo especial de usuários de drogas que sofreram trauma físico associado à intoxicação por abuso, fosse por envolvimento em situações de violência, acidente ou queda.

A idade dos usuários de drogas variou entre 20 e 65 anos, com média de 40,1 anos (DP $\pm 12,1)$, eram, em sua maioria, do sexo masculino $(28-93,3 \%)$, solteiros $(22-75,9 \%)$ e 
desempregados ( $15-51,7 \%)$. O tempo de uso de drogas variou de um a 54 anos, com média de 20,8 anos. Homens apresentam maior uso na vida e maior dependência de álcool e de outras drogas do que mulheres em todas as faixas etárias, independente da droga de abuso que utilizam $^{(2,6,17)}$. Porém, em relação à faixa etária, no presente estudo, verificou-se divergência em relação ao padrão etário nacional, que é de 18 a 34 anos, possivelmente relacionada ao tempo de uso da(s) droga(s), que também não confere com a média estabelecida no inquérito de base populacional, que é de 13 anos $^{(17)}$.

O álcool foi a droga referida pela maioria dos eventos sentinela à internação hospitalar (28 - 93,3\%), confirmada por critérios clínicos ou laboratoriais, mas 13 (44, 8\%) faziam uso associado de várias drogas, sendo que metade fazia uso diário da droga de abuso e realizava manobras ilícitas para aquisição desta. O II Levantamento Nacional de Álcool e Drogas apontou que, aproximadamente, 5,7\% da população brasileira são dependentes de álcool e/ou maconha e/ou cocaína, com mais de oito milhões de pessoas usuárias de drogas de abuso e 30 milhões de pessoas convivendo com um usuário de drogas ${ }^{(2,17)}$. As características sociodemográficas dos eventos sentinela, do presente estudo, acompanharam o perfil nacional de usuários de drogas de abuso quanto ao sexo, conjugalidade e ocupação, encontrados em outros estudos $^{(6,17)}$.

O padrão de consumo de drogas é um fenômeno dinâmico, com usuários experimentando diferentes combinações de drogas, misturando as lícitas e ilícitas, além de várias formas de consumo. O uso de várias substâncias, simultaneamente ou em sequência, está aumentando em vários países, principalmente a combinação de álcool com drogas ilícitas ${ }^{(18)}$. No presente estudo, o padrão do uso de drogas acompanhou a tendência referida e caracterizou-se pelo uso múltiplo ou poliuso de drogas.

Este estudo apontou frequência do uso diário de drogas e manobras ilícitas para aquisição destas. Esse quadro configura-se como situação de extrema vulnerabilidade e essas condições são marcadoras da gravidade do abuso de drogas, onde o indivíduo se expõe a fatores que contribuem para as situações de violência no cotidiano familiar ${ }^{(19)}$. Essas práticas são apontadas, por estudos, como indicadores de sofrimento familiar, e este se complica com o avanço da dependência pelo rompimento dos vínculos familiares, fator este que favorece o uso diário de drogas e as manobras ilícitas ${ }^{(2,3,19)}$.

Já foi verificado que, quanto maior a vulnerabilidade da família em relação às condições de escolaridade, maior é a vulnerabilidade com relação ao acesso ao trabalho e renda, o que é elencado como fator de risco para a iniciação do uso de drogas na família, com menor disponibilidade financeira vista como uma das maiores contribuições para a vulnerabilidade social e abuso de drogas. Sabe-se que, em consequência do consumo de drogas, dificilmente o usuário se mantém empregado, o que o leva à prática de pequenos delitos dentro do lar e ocasiona danos ao patrimônio na sociedade ${ }^{(3)}$.

A maioria dos familiares entrevistados era mulheres (26-86,7\%), principalmente mães do usuário de droga (14 - 46,7\%), e sete delas se encontravam em situação de "chefe da família". A escolaridade média era de 7,5 anos de estudo (DP $\pm 4,8) ; 16$ (53,3\%) autorreferiram alguma doença ou problema de saúde crônico, principalmente hipertensão arterial sistêmica, depressão e diabetes mellitus.

Os dados de caracterização dos familiares entrevistados corroboram com estudos nacionais, que constataram percentuais semelhantes de pessoas disponíveis a informar sobre o problema das drogas em suas famílias. Geralmente, são mães que convivem cotidianamente com 0 impacto negativo do uso de drogas na família, com a sobrecarga de cuidar do filho com comportamento aditivo ${ }^{(3,7,19)}$.

Quanto aos relatos de doenças crônicodegenerativas não transmissíveis e transtornos mentais comuns, presentes em mais da metade dos entrevistados, o consumo aditivo no ambiente familiar apresenta-se como causa e fator contribuinte para $\mathrm{o}$ adoecimento e sobrecarga de seus membros, principalmente os familiares mais próximos como mães e esposas $^{(3,19)}$. Familiares de usuários de drogas apresentam significativamente, mais sintomas físicos crônicos e queixas de sofrimento mental e maiores dificuldades psicossociais que a média da população, independente da droga utilizada ${ }^{(1)}$.

A partir da aplicação da Escala de Hopefulness-hopelessness, gerou-se a Tabela 1, na qual se pode observar a média de cada um dos dez itens que a compõem, bem como o desviopadrão e a mediana. A média de esperança 
encontrada foi de $28,81( \pm 2,83)$ e a mediana de 30,0 .

Tabela 1 - Estatística descritiva dos escores da Escala de Esperança de Hopefulness-hopelessness de 29 familiares dos eventos sentinela. Paraná, 2014.

\begin{tabular}{|c|c|c|}
\hline Afirmativas & Média ( $\pm \mathrm{DP})$ & Mediana \\
\hline 1 - Estou começando a prever novo futuro $(+)^{*}$ & $3,79( \pm 0,29)$ & 5,00 \\
\hline 2 - Acredito que fora disso alguma coisa boa virá $(+)$ & $4,07( \pm 0,28)$ & 5,00 \\
\hline 3 - As coisas estão começando a melhorar $(+)$ & $3,17( \pm 0,31)$ & 4,00 \\
\hline 4 - Estou começando a ter de volta a pessoa que conheci $(+)$ & $2,90( \pm 0,30)$ & 3,00 \\
\hline 5 - Me sinto mais positivo sobre as coisas $(+)$ & $3,41( \pm 0,32)$ & 4,00 \\
\hline 6 - Estou temoroso sobre como meu familiar vai prosseguir $(-)^{*}$ & $1,72( \pm 0,27)$ & 1,00 \\
\hline 7 - Meu familiar não está encarando as coisas com seriedade (-) & $2,34( \pm 0,30)$ & 1,00 \\
\hline 8 - Estou pessimista em relação ao futuro imediato (-) & $2,59( \pm 0,30)$ & 2,00 \\
\hline 9 - Nunca haverá nenhuma mudança, meu familiar está no mesmo lugar (-) & $3,34( \pm 0,27)$ & 4,00 \\
\hline 10 - Temo que meu familiar vá beber e se drogar até o fim (-) & $1,48( \pm 0,19)$ & 1,00 \\
\hline Total & $28,81( \pm 2,83)$ & 30,0 \\
\hline
\end{tabular}

*(+) itens positivos. ${ }^{*}(-)$ itens negativos- escores invertidos.

Dentre os dez itens da escala, o item número 2 apresentou o escore de esperança mais alto (média 3,65), sendo que 68,9\% dos respondentes concordavam $(13,8 \%)$ ou concordavam completamente $(55,1 \%)$ com a afirmativa 'acredito que fora disso alguma coisa boa virá'. Por outro lado, o item com escore mais baixo foi o de número 10 (média 1,48), indicando que $89,7 \%$ concordavam ou concordavam completamente com a afirmativa 'temo que meu familiar vá beber e se drogar até o fim', com percentuais de 13,8 e $75,9 \%$, respectivamente (Tabela 1).

Estudos que avaliaram a esperança de familiares cuidadores foram realizados em diferentes populações, como familiares cuidadores de doentes crônicos ${ }^{(9)}$ e de portadores de deficiência cognitiva e esquizofrenia ${ }^{(11,20)}$, porém apenas o Levantamento Nacional de
Famílias de Dependentes Químicos - LENAD Família - avaliou a esperança de familiares de usuários de drogas ${ }^{(7)}$.

O LENAD Família, estudo nacional que teve como informantes familiares de usuários de álcool e de usuários de cocaína e/ou crack em tratamento para o uso e drogas, avaliou a esperança dos familiares por meio da Escala de Hopefulness-hopelessness ${ }^{(15),}$ e identificou um nível médio de esperança de $36,5^{(7)}$. A média de esperança do presente estudo $(28,8)$ foi inferior à encontrada no LENAD Família, e esse resultado pode ser relacionado ao tempo médio de uso de drogas pelos usuários - 13 anos no LENAD Família e 20,8 anos neste estudo, além disso, no período da pesquisa, nenhum dos usuários de drogas estava recebendo tratamento para cessar o uso de drogas.

Tabela 2 - Sumário estatístico de variáveis sociodemográficas e escore de Escala de Hopefulnesshopelessness de 29 familiares dos eventos sentinela. Paraná, 2014.

\begin{tabular}{|c|c|c|c|c|}
\hline Variável & Categorias & $\mathrm{n}(\%)$ & Mediana & $p^{*}$ \\
\hline \multirow{2}{*}{ Tempo de uso de droga de abuso } & Até 24 anos & $13(44,8)$ & 32 & \multirow{2}{*}{0,011} \\
\hline & 25 anos ou mais & $16(55,2)$ & 21,5 & \\
\hline \multirow{2}{*}{ Poliuso de droga de abuso } & Sim & $13(44,8)$ & 18 & \multirow{2}{*}{$<0,001$} \\
\hline & Não & $16(55,2)$ & 37 & \\
\hline \multirow{2}{*}{ Idade do usuário } & Até 46 anos & $15(51,7)$ & 32 & \multirow{2}{*}{0,038} \\
\hline & De 47 a 65 anos & $14(48,3)$ & 19,5 & \\
\hline \multirow{2}{*}{ Idade do familiar } & Até 53 anos & $15(51,7)$ & 31 & \multirow{2}{*}{0,616} \\
\hline & Mais de 53 anos & $14(48,3)$ & 35,5 & \\
\hline \multirow{2}{*}{ Religião } & Praticante & $19(65,5)$ & 33 & \multirow{2}{*}{0,012} \\
\hline & Não praticante & $10(34,5)$ & 15,5 & \\
\hline \multirow[t]{2}{*}{ Escolaridade } & $<8$ anos & $16(55,2)$ & 31,5 & \multirow[t]{2}{*}{0,779} \\
\hline & $>8$ anos & $13(44,8)$ & 31 & \\
\hline
\end{tabular}

\footnotetext{
*Teste de Mann-Whitney.
} 
Em relação aos fatores que influenciaram a esperança familiar, o tempo de uso de droga de abuso superior a 24 anos, poliuso de droga de abuso pelos usuários e idade do usuário superior a 46 anos obtiveram correlação negativa em relação ao nível de esperança, ou seja, apresentaram mediana de esperança significativamente menor. No entanto, a prática religiosa pelo familiar se mostrou fator contribuinte na esperança deste, apresentando mediana de esperança significativamente maior que a dos nãos praticantes. $A$ idade e a escolaridade do familiar não foram estatisticamente associadas aos escores de esperança das famílias (TABELA 2).

O tempo prolongado de uso de drogas foi estatisticamente associado a um nível mais baixo de esperança dos familiares bem como à presença do poliuso de drogas. Estudos apontam que a exposição a essas trajetórias de riscos agrava as condições de vulnerabilidade das famílias, uma vez que estas vivenciam longos períodos de sobrecarga, tais como, alterações na dinâmica familiar, problemas econômicos, atos ilícitos envolvendo agressões e roubos, adoecimento físico e psíquico de alguns membros da família que vivenciam situações de violência e estresse, gerando sentimentos de impotência, tristeza profunda e desesperança diante do comportamento aditivo, ou seja, intenso sofrimento familiar ${ }^{(3,16,21)}$.

Estudo realizado em Curitiba-PR apontou que $65 \%$ dos dependentes químicos em tratamento encontravam-se na faixa etária inferior a 40 anos, principalmente concentrada entre 20 a 29 anos $(30,8 \%)^{(22)}$. Dessa forma, as famílias do presente estudo, que convivem com usuários com idades superiores, devem cultivar, em maior escala, sentimentos de desmotivação, descrença e incertezas em relação ao tratamento e cessação do uso de drogas, o que implica correlação negativa de esperança familiar.

A maior pontuação na escala foi a da afirmativa 'acredito que fora disso alguma coisa boa virá', indicando que os familiares ainda mantêm algum sentimento de esperança em relação ao futuro. Essa afirmativa, mesmo diante das incertezas e da difícil situação atual, pode estar relacionada à espiritualidade dos familiares, visto que o estudo apresentou correlação positiva com a religião, ou seja, familiares que vivenciam a prática religiosa, obtém níveis de esperança significativamente mais altos.
Ainda em relação à prática religiosa com a esperança, o resultado desta pesquisa corrobora com estudo que avaliou a esperança em cuidadores de idosos com algum grau de dependência, apontando que a espiritualidade se configura como fator protetor da esperança e que, quanto maior o nível de espiritualidade, menor a chance de os cuidadores se sentirem desesperançosos ${ }^{(9)}$. Nas relações entre dependentes químicos e seus familiares, apontou-se que a religião é importe alicerce para o enfrentamento do sofrimento de famílias que convivem com comportamento aditivo, auxiliando na resiliência perante as dificuldades acrescidas no cotidiano familiar ${ }^{(21)}$.

No entanto, a menor pontuação na escala foi da afirmativa 'temo que meu familiar vá beber e se drogar até o fim', expressando o medo da família em relação ao futuro do seu familiar, principalmente por desacreditar que este possa cessar o uso e drogas. Estudo que teve como objetivo identificar o modo como a codependência é expressa no grupo de familiares de dependentes químicos, apontou que o medo é um dos sentimentos mais presentes e expressos na vida dessas famílias e está relacionado, principalmente, às situações de violência, de abandono, de recaídas e de que aconteça "algo ruim" no futuro do dependente químico ${ }^{(23)}$.

Os familiares vivenciam a luta e enfrentamento cotidiano, tanto pelo convívio direto com o usuário quanto pelos sentimentos de incerteza e insegurança em relação ao futuro ${ }^{(3,7,23)}$. A convivência, muitas vezes, se manifesta na forma de sintomas físicos e psicológicos, tornando esses indivíduos vulneráveis e com necessidades de atenção e cuidados específicos. O conhecimento da esperança dos cuidadores do usuário de drogas é importante para o planejamento de políticas de saúde pública visando ao amparo dessa população, de maneira a fortalecer a esperança da família.

\section{CONCLUSÃO}

Conclui-se que o perfil dos eventos sentinela acompanhou o descrito na literatura, em sua maioria do sexo masculino, solteiros e desempregados, porém, em relação ao padrão etário e tempo de uso de droga, o presente estudo apresentou índices superiores aos da literatura e apontou famílias com percentuais sociodemográficos semelhantes ao perfil nacional de famílias pertencentes às camadas populares. 
Chama atenção, neste estudo, o apontamento de diversos problemas de saúde entre os familiares que podem estar associados à sobrecarga da convivência com o usuário de drogas.

Este estudo tornou possível a mensuração da esperança em uma amostra composta por familiares de usuários de drogas. Em comparação com o estudo brasileiro que avaliou a esperança em famílias de usuários de drogas, observou-se que a média de esperança apresentada pelas famílias foi menor.

Mesmo as famílias obtendo uma pontuação otimista em relação ao futuro de seu familiar, acreditando que 'fora disso alguma coisa boa virá', uma pontuação expressiva foi evidenciada em relação ao temor, que as famílias apresentam, de que os usuários de drogas se droguem até o fim. Observou-se que o tempo prolongado de uso de drogas, o poliuso de drogas, a idade dos usuários e a prática religiosa pelos familiares influenciaram significativamente no nível de esperança das famílias com comportamento aditivo.

A esperança é um importante sentimento para a colaboração da resiliência das famílias que passam por sofrimento familiar, sendo um dos aspectos centrais no cuidado de enfermagem, contribuindo para se lidar com situações de crise, para a manutenção da qualidade de vida e para a promoção da saúde. $O$ presente estudo mostra a necessidade do planejamento de políticas de saúde pública visando ao amparo de famílias que convivem com usuários de drogas.

\section{REFERÊNCIAS}

1- Maciel SC, Melo JRF, Dias CCV. Depressive symptoms in family members of drug-addicts. Psicol: teor prát. 2014;16(2):18-28. DOI: 10.15348/1980-6906/psicologia.v16n2p18-28

2- Sakiyama HMT, Padin MFR, Canfield M, Laranjeira R, Mitsuhiro SS. Family members affected by a relative's substance misuse looking for social support: who are they? Drug Alcohol Depend. 2015;147:276-9. DOI: 10.1016/j.drugalcdep.2014.11.030

3- Reis LM, Oliveira MLF. Social vulnerability in families living with long-term addictive behavior. Acta Paul Enferm. 2017;30(4):412-9. DOI: 10.1590/1982-0194201700061

4- Watson J, Toner P, Day E, Back D, Brady L-M, Fairhurst $C$, et al. Youth social behaviour and network therapy (Y-SBNT): adaptation of a family and social network intervention for young people who misuse alcohol and drugs - a randomised controlled feasibility trial. Health Technol Assess. 2017;21(15):1-260. DOI: 10.3310/hta21150

5- Goings TC, Salas-Wright CP, Howard MO, Vaughn MG. Substance use among bi/multiracial youth in the United States: profiles of psychosocial risk and protection. Am J Drug Alcohol Abuse 2018;44(2):206-14. DOI: 10.1080/00952990.2017.1359617

6- Seleghim MR. A trajetória de usuários de crack para a situação de rua na perspectiva de familiares [on-line]. [tese]. Ribeirão Preto: Universidade de São Paulo; 2016.

7- Laranjeira RR, organizador. Levantamento nacional de familias dos dependentes quimicos. São Paulo: INPAD; 2014.

8- Du H, King RB. Placing hope in self and others: Exploring the relationships among self-construals, locus of hope, and adjustment. Pers Individ Dif. 2013;54(3):332-7.

DOI:

10.1016/i.paid.2012.09.015

9- Balsanelli ACS, Grossi SAA, Herth K. Avaliação da esperança em pacientes com doença crônica e em familiares ou cuidadores. Acta Paul Enferm. 2011;24(3):354-8. DOI: $10.1590 /$ S010321002011000300008

10- Hernandez M, Barrio C, Gaona L, Helu-Brown P, Hai A, Lim C. Hope and Schizophrenia in the Latino Family Context. Community Ment Health J. 2019;55(1):42-50. DOI: 10.1007/s10597-0180354-5

11- Wakiuchi J, Marchi JA, Norvila LS, Marcon SS, Sales CA, Wakiuchi J, et al. Esperança de vida de pacientes com câncer submetidos à quimioterapia. Acta Paul Enferm. 2015;28(3):202-8. DOI: $\quad \underline{10.1590 / 1982-}$ $\underline{0194201500035}$

12- Rutstein DD, Berenberg W, Chalmers TC, Child CG, Fishman AP, Perrin EB, et al. Measuring the quality of medical care. A clinical method. N Engl J Med. 1976;294(11):582-8. DOI: 10.1056/NEJM197603112941104

13- Santana CJ, Silvino MCS, Rosa NM, Almeida EG, Reis LM, Oliveira MLF. Potentiality of a sentinel event for epidemiological surveillance of drug abuse. J Nurs UFPE 2014 [citado em 1 abr 2019]; 8(12):321-7. Disponível em: https://periodicos.ufpe.br/revistas/revistaenferm agem/article/view/10181

14- Teixeira TCA, Cassiani SHB, Teixeira TCA, Cassiani SHB. Análise de causa raiz de acidentes por quedas e erros de medicação em hospital. Acta Paul Enferm. 2014;27(2):100-7. DOI: 10.1590/1982-0194201400019

15- Orford J, Templeton L, Velleman R, Copello 
A. Family members of relatives with alcohol, drug and gambling problems: a set of standardized questionnaires for assessing stress, coping and strain. Addiction 2005; 100(11):1611-24. DOI: 10.1111/j.1360-0443.2005.01178.x

16- Sola V, Sakiyama HMT, Padin MRF, Canfield $M$, Bortolon $C B$, Laranjeira $R$, et al. Measuring stress, coping, strain and hopefulness of Brazilian family members of substance misusers: Factor structure of a set of measures. J Subst Use 2019;24(2):130-9.

$10.1080 / 14659891.2018 .1523963$

17- Instituto Nacional de Ciência e Tecnologia para Políticas Públicas de Álcool e Outras Drogas (INPAD). II Levantamento Nacional de Álcool e Drogas (LENAD): relatório 2012. São Paulo: INPAD; 2014.

18- World Health Organization (WHO). Global status report on alcohol and health 2014. Geneve: World Healt Organization; 2014.

19- Santana CJ, Oliveira MLF. Efeitos do envolvimento com drogas na vida de familiares de usuários por longo período. Rev Rene 2017 [citado em 1 abr 2019]; 18(5):671-8. Disponível em:

http://periodicos.ufc.br/rene/article/view/30843 771502

20- Hunsaker AE, Terhorst L, Gentry A, Lingler $\mathrm{JH}$. Measuring hope among families impacted by cognitive impairment. Dementia 2016;15(4):596608. DOI: $10.1177 / 1471301214531590$

21- Takahara AH, Furino $\mathrm{V}$, Marques AC, Zerbetto S, Furino F. Relações familiares, álcool e outras drogas: uma revisão integrativa. Rev APS 2017 [citado em 01 abr 2019]; 20(3):434-43. Disponível em: http://ojs2.ufjf.emnuvens.com.br/aps/article/vie $\mathrm{w} / 15999$

22- Nimtz MA, Tavares AMF, Maftum MA, Ferreira ACZ, Borba LO, Capistrano FC. The impact of drug use on the family Relationships of drug addicts. Cogitare Enferm. 2014 [citado em 02 dez 2018]; 19(4):667-72. DOI: 10.5380/ce.v19i4.35721

23- Moraes LMP, Braga VAB, Souza AMA, Ória MOB. Expression of co-dependence among relative of chemically dependent patients. Rev Min Enferm. 2009 [citado em 01 abr 2019]; 13(1):34-42. Disponível em: http://www.reme.org.br/content/imagebank/pdf v13n1a06.pdf
NOTA: O presente trabalho foi realizado com apoio da Coordenação de Aperfeiçoamento de Pessoal de Nível Superior - Brasil (CAPES) - Código de Financiamento 001.

Recebido em: 22/12/2018

Aprovado em: 16/08/2019

Endereço de correspondência: Indianathan de Kassia Santana Elvira Avenida Horácio Racanello Filho, no 5450, apartamento 904 - Zona 7 CEP: 87020-035 -Maringá/PR - Brasil

E- mail: indianathan 19@hotmail.com 